\title{
Über quadratische Formen mit reellen Koeffizienten.
}

\author{
Von Ernst Fischer in Brünn.
}

Einleitung.

a) Die Klassifikation der quadratisehen Formen

$$
f(x)=\sum_{1}^{n} a_{i k} x_{i} x_{k}
$$

von $n$ Unbestimmten $x_{1}, x_{2}, \ldots x_{n}$ mit reellen Koeffizienten $a_{i k}=a_{k i}$ ist aus zwei verschiedenen Gesichtspunkten vorgenommen worden, welche hier kurz als der algebraische und der analytische unterschieden werden mögen.

Die algebraische Einteilung rechnet zwei Formen dann und nur dann in dieselbe Klasse, wenn sie gegenseitig auseinander durch linearhomogene $\mathrm{Tr}$ an $\mathrm{s}$ ormation mit reellen Koeffizienten hervorgehen. Es bilden dann die Formen:

$x_{1}^{2}+x_{2}^{2}+\cdots+x_{\pi}^{2}-x_{\pi+1}^{2} \cdots x_{\pi+2}^{2}-\cdots-x_{v+\nu}^{2}\left(\begin{array}{c}\pi, \nu=0,1,2, \ldots n \\ \pi+\nu \leqq_{n}\end{array}\right)$ ein volles System von Klassenrepräsentanten, so daß die Klassen durch die Zahlenpaare $(\pi, v)$ charakterisiert erscheinen. Unter dem einer einzelnen Form $f$ zugehörigen Zahlenpaare $(\tau, v)$ werde das Zahlenpaar der Klasse verstanden, in welcher $f$ enthalten ist.

Aus dem analytischen Gesichtspunkte dagegen werden die Vorzeichen der Funktionswerte in Betracht gezogen, welche die Funktion $f$ annimmt, während ihre Variabelen $x_{1}, x_{2}, \ldots x_{n}$ das Gebiet der reellen Zahlen durchlaufen. Danach werden dreierlei Formen unterschieden: positiv definite, indefinite, negativ definite.

Diese beiden Einteilungen hängen aber mit einander zusammen. Die positiv definiten ${ }^{\text {) }}$ Formen sind die Formen der Klasse $(\pi=n, \nu=0)$, die negativ definiten die der Klasse $(\pi=0, \nu=n)$, die indefiniten die der übrigen Klassen. So stellt sich die algebraische Einteilung - der Sache nach - als eine Verfeinerung der analytischen dar.

Hierzu ist aber Folgendes zu bemerken. Der analytische Gesichtspunkt, die Funktionswerte und ihre Vorzeichen zu beachten,

1) Die Benennung "definit" wird hier stets in dem engeren Sinne gebraucht, wonach $f=0$ nur für $x_{1}=x_{2}=\cdots=x_{n}=0$ stathaft ist. 
ist nur ein ganz allgemeiner, welchem bestimmte Einteilungsgründe erst entnommen werden müssen. Ein solcher bestimmter Einteilungsgrund ist der, welcher zu der erwähnten Dreiteilung geführt hat: man untersucht, welche Vorzeichen die Funktion $f$ überhaupt anzunehmen vermag. Man kann aber dem allgemeinen analytischen Gesichtspunkte sebr wohl auch andere und feinere Einteilungsgründe entnehmen, indem man beachtet, in welcher Weise im Gebiete der reellen Veränderlichen $x_{1}, x_{2}, \ldots x_{n}$ die Stellen verteilt sind, an welchen $f>0$, und diejenigen, an welchen $f<0$ ist. Und es wäre zu erwägen, ob nicht eine dieser feineren analytischen Einteilungen sich mit der algebraischen der Sache nach - genau decken mag.

Daß dies wirklich der Fall ist, leuchtet ein, wenn man die oben angeführten Klassenrepräsentanten der algebraischen Einteilung ins Auge faßt: sie weisen deutlich genug darauf hin, wie man den Begriff des einer Form $f$ zugehörigen Zahlenpaares $(\pi, v)$ analytisch, aus Vorzeichen von Funktionswerten, verstehen kann. $\left.{ }^{2}\right)$ ( $\left.\$ \$ 1,2.\right)$

Insoferne dabei die Zahlen $\pi$ und $v$ an der einzelnen Form konstruiert werden, gewinnt man eine willkommene Ergänzung der algebraischen Auffassung. War nämlich die algebraische Klassifikation gegründet auf eine Beziehung zwischen $z w$ ei Formen derselben Klasse, so dient als analytischer Einteilungsgrund eine Eigenschaft der einzelnen Form, in welcher die Formen einer Klasse übereinstimmen. Nun vermag freilich auch die algebraische Theorie in ihrer weiteren Entwicklung die Klassen durch Eigenschaften der einzelnen Form zu charakterisieren, und dies in vollendeter Übersichtlichkeit, aber, wie ich meine, nie durch Eigenschaften von selbständigem Interesse, d. h. durch Eigenschaften, welche auch dann bedeutsam erscheinen müßten, wenn ihr Zusammenhang mit der Klassifikation nach linearer Transformierbarkeit noch nicht bekannt wäre.

b) Es ist sodann interessant, mit einer derart verfeinerten analytischen Auffassung auch dem weiteren Ausbau der algebraischen Theorie zu folgen:

Die Frage nach der Klasse $(\pi, v)$, welcher die Form $f$ angehört, ist enthalten in der Frage nach den Klassen, durch welche die Form $f-t F^{r}$ hindurchgeht, während $t$ das Gebiet der reellen Zahlen durchläuft; dabei bedeute $F(x)=\sum_{1}^{n} A_{i k} x_{i} x_{k}$ irgend eine positiv definite ${ }^{1}$ ) Form. Auf diese Frage antwortet die algebraische Theorie durch den Satz, daß sich die Form $f-t F$ durch linearhomogene Transformation mit nichtverschwindender Determinante und ron $t$ unabhängigen reellen Koeffizienten in die Gestalt

2) Es handelt sich also z. B. darum, die reellkollineare Klassifikation der Flächen 2. Ordnung za gründen auf eine anschaulich-geometrische Bedeutung der Zahlen $\pi$, $\vee$ selbst; dies gelingt ohne Schwierigkeit, s. unten Fußnote 7). 
$\left(a_{1}-t\right) x_{1}^{2}+\left(a_{2}-t\right) x_{2}^{2}+\cdots+\left(a_{n}-t\right) x_{n}^{2}$ überführen läßt, wo $a_{1}$, $a_{2}, \ldots a_{n}$ die Wurzelu der Determinantengleichung $\left|a_{i k}-t A_{i k}\right|=0$ bedeuten. Danach sind die der Form $f-t F$ zugehörigen Zahlen $\pi(t), v(t)$ identisch mit der Anzahl derjenigen unter den Wurzeln $a_{1}, a_{2}, \ldots a_{n}$, welche größer resp. kleiner sind als $t$. Und $a_{1}, a_{2}, \ldots a_{n}$ sind diejenigen Werte von $t$, bei deren Überschreitung die Form $f-t F$ aus einer Klasse in eine andere übertritt.

Von diesen Tatsachen fällt in das Bereich der bisherigen analytischen Auffassung bloß die Bedeutang der äußersten Wurzeln $a_{1}$ und $a_{n}$ (wo $a_{1} \geq a_{2} \geq \cdots \geq a_{n}$ angenommen) als Maximum and Minimum derjenigen Werte $t$, für welche die Form $f-t F$ indefinit ist. In das Bereich der verfeinerten analytischen Auffassung fallen sie aber in ihrer Gänze. Es muß daher gelingen, sie auch unabhängig von der algebraischen Theorie aus dem analytischen Ansatze zu entwickeln. ( $\$ \$ 3-6$.) Die Deduktion wird das algebraische Hilfsmittel der linearen Transformation zu vermeiden haben, hingegen von Stetigkeitsbetrachtungen Gebrauch machen dürfen.

Die Rolle, welche, wie erwähnt, $a_{1}$ und $a_{n}$ in der bisherigen analytischen Auffassung spielen, kann ersichtlich auch so gekennzeichnet werden: $a_{1}$ und $a_{n}$ sind das Maximum und Minimum der Werte $t$, welche der Quotient $\frac{f}{F}$ annimmt, wenn $\left(x_{1}, x_{2}, \ldots x_{n}\right)$ alle reellen Wertsysteme außer $(0,0, \ldots 0)$ durchläuft, $\left.{ }^{3}\right)$ oder aber der Werte, welche $f$ auf der aus den reellen Lösungen der Gleichung $F=1$ bestehenden Mannigfaltigkeit annimmt. ${ }^{4}$ ) In entsprechender Weise mub aus der verfeinerten analytischen Auffassung eine Deutung der sämtlichen Wurzeln $a_{1}, a_{2}, \ldots a_{n}$ als Maxima und Minima von gewissen Funktionswerten erwachsen. ( $\$ 7$.

In diesem Sinne soll nun im Folgenden der Versuch unternommen werden, durch Wiederaufnahme des alten analytischen Ansatzes der algebraischen Theorie eine analytische Nachbildung an die Seite zu stellen. ${ }^{5}$ ) Denn man kann von den hohen Vorziugen

3) Geometrisch ausgedrückt : Da proportionale Wertsysteme der Variabelen Funktionswerte vom selben Vorzeichen ergeben, deute man $x_{1}, x_{2}, \ldots x_{n}$ als homogene Koordinaten eines Raumes von $n-1$ Dimensionen and definiere den Wert $f\left(x_{1}: x_{2}: \cdots: x_{n}\right)$, welchen die Form im Punkte $\left(x_{1}: x_{2}: \cdots: x_{n}\right)$ annimmt, durch $f\left(x_{1}: x_{2}: \ldots: x_{n}\right)=\frac{f\left(x_{1}, x_{2}, \ldots x_{n}\right)}{F\left(x_{1}, x_{2}, \ldots x_{n}\right)}$. Dann handelt es sich um das Maximum. und Minimum der Werte, welche die Form im Raume annimmt. $f-t F=0$ bedeutet dann die "Niveaufläche" $f\left(x_{1}: x_{2}: \cdots: x_{n}\right)=t$, und $t=a_{1}, t=a_{n}$ geben die äußersten Niveauflächen, welche noch reelle Punkte besitzen.

4) Beweis mit den Mitteln der Lehre von Maximum und Minimun bei C. Jord a n, cours d'analyse, 2. éd., t. I, p. 392 und O. Stolz, Diff.-Rechn. I, p. 248.

5) Der Aufbau der beiden Theorien gestaltet sich nicht parallel. Die Sätze über die Gleichung $\left|a_{i k}-t A_{i k}\right|=0$, welche unten (Ende des $\$ 6$ ) C. und $D$. heißen, ergeben sich in der analytischen Theorie zuallerletzt, als Nebenresultate. 
einer Theorie durchdrungen sein, und doch noch den Wunseh haben, zur Ergänzung den Gegenstand auch von einer anderen Seite zu beleuchten.

\section{§ I. Der Positivrang $\pi$ und der Negativrang $\nu$ einer quadratischen Form mit reellen Koeffizienten.}

Es seien $x_{1}, x_{2}, \ldots x_{n}$ reelle unbeschränkt veränderliche Größen.

Ein Inbegriff ron Wertsystemen $(x)=\left(x_{1}, x_{2}, \ldots x_{n}\right)$ dieser Größen werde ein ebenes Gebilde genannt, wenn er aus den sämtlichen reellen Lösungen eines linearhomogenen Gleichungssystems mit reellen Koeffizienten besteht; oder, um "innere" Eigenschaften zu Grunde zu legen, wenn:

$1^{\circ}$ neben $(x)=\left(x_{1}, x_{2}, \ldots x_{n}\right)$ auch allemal $\lambda(x)=\left(\lambda x_{1}, \lambda x_{2}, \ldots \lambda x_{n}\right)$ ihm angehört, wo $\lambda$ jede beliebige reelle Zahl,

$2^{0}$ neben $(x)=\left(x_{1}, x_{2}, \ldots x_{n}\right)$ und $\left(x^{\prime}\right)=\left(x_{1}^{\prime}, x_{2}^{\prime}, \ldots x_{n}^{\prime}\right)$ auch allemal $\left(x+x^{\prime}\right)=\left(x_{1}+x_{1}^{\prime}, x_{2}+x_{2}^{\prime}, \cdots x_{n}+x_{n}^{\prime}\right)$ ihm angehört.

Gibt es in einem ebenen Gebilde ein System von $k$, aber kein System von $k+1$ linearunabhängigen Wertsystemen, so werde $k$ als die Stufenzahl des ebenen Gebildes bezeichnet $(0 \leq k \leq n)$.

Nun sei $f(x)=f\left(x_{1}, x_{2}, \ldots x_{n}\right)$ eine quadratische Form mit reellen Koeffizienten.

Gibt es ein ebenes Gebilde $\pi^{\text {ter }}$ Stufe, jedoch keines $\pi+1^{\text {ter }}$ Stufe, auf welchem mit alleiniger Ausnahme des Wertsystems $(0)=(0,0, \ldots 0)$ stets $f>0$ ist, so soll $\pi$ der Positivrang der Form $f$ heißen $(0 \leq \pi \leq n)$.

Gibt es ein ebenes Gebilde $\nu^{\text {ter }}$ Stufe, jedoch keines $\nu+1^{\text {ter }}$ Stufe, auf welchem mit alleiniger Ausnahme des Wertsystems (0) stets $f<0$ ist, so soll v der $\mathrm{N}$ e gativrang der Form $f$ heiben $\left.^{6}\right),{ }^{7}$ ) $(0 \leq v \leqq n)$.

\section{\$ 2. Zusammenhang mit der algebraischen Definition von $\pi$ und $v$.}

Das Trägheitsgesetz der quadratischen Formen nimmt in unserem Zusammenhange die folgende Gestalt an:

Man führe durchlinearhomogene Transformation mit nichtverschwindender Determinante und reellen

b) Daß die Zahlen $\pi$ und $\vee$ gegenüber linearhomogener Transformation mit nichtverschwindender Determinante und reellem Koeffizienten invariant sind, liegt bei dieser Art der Definition unmittelbar auf der Hand.

7) Ist $n=4$ und sind $x_{1}, x_{2}, x_{3}, x_{4}$ homogene Koordinaten der Punkte des Raumes, so teilt die Fläche 2. Ordnung $f=0$ den ganzen Raum in zwei Ränme, die durch $f>0$ und $f<0$ unterschieden werden. Die Zahlen $\pi, v$ enthalten dann Aussagen über die Gestalt dieser Räume. Bei einer elliptischen Fläche z, B, hat in dem einen Raume eine Ebene Platz, im anderen von ebenen Gebilden nur ein Punkt; dem entsprechend hat von den Zahlen $\tau$ und $v$ eine den Wert 3 , eine den Wert 1. Bei einer hyperbolischen Fläche hat in jedem der beiden Räume eine Gerade Platz, aber keine Ebene: $\pi=2, \nu=2$. Entsprechend bei den Kegeln, u. s. w. 
Koeffizienten neue Variabele $x_{1}^{\prime}, x_{2}^{\prime}, \ldots x_{n}^{\prime}$ ein. Wird hiebei:

$$
f\left(x_{1}, x_{2}, \ldots x_{n}\right)=f_{1}\left(x_{1}^{\prime}, x_{2}^{\prime}, \ldots x_{\pi x}^{\prime}\right)-f_{2}\left(x_{\pi+1}^{\prime}, x_{\pi+2}^{\prime}, \ldots x_{\pi+v}^{\prime}\right),
$$

wo $f_{1}$ und $f_{2}$ positiv definite Formen ihrer $\pi$ resp. Variabelen sind, so sind die Zahlen $\pi$ und $\nu$ der Positivrang und Negativrang der Form $f\left(x_{1}, x_{2}, \ldots x_{n}\right)$. Beweis: Durch das Gleichungssystem

$$
x_{r+1}^{\prime}=0, x_{\pi+2}^{\prime}=0, \ldots x_{n}^{\prime}=0
$$

wird ein ebenes Gebilde $\pi^{\text {ter }}$ Stufe definiert. Auf demselben ist $f(x)=f_{1}\left(x_{1}^{\prime}, x_{2}^{\prime}, \cdots x_{x}^{\prime}\right)$, also stets $f(x) \geq 0$, und nur dann $f(x)=0$, wenn

$$
x_{1}^{\prime}=0, x_{2}^{\prime}=0 \ldots x_{\pi}^{\prime}=0
$$

ist; in diesem letzteren Falle ist aber wegen (a) und (b) auch $(x)=0$. Damit ist die Existenz eines ebenen Gebildes $\pi^{\text {ter }}$ Stufe nachgewiesen, auf welchem durchaus $f>0$, mit alleiniger Ausnahme des Wertsystems (0).

Angenommen nun, es gebe auch ein ebenes Gebilde $\pi+1^{\text {ter }}$ Stufe $(E$, auf welchem durchaus $f>0$ mit alleiniger Ausnahme des Wertsystems (0). Dann sei (5* das ebene Gebilde $n-\pi^{\text {ter }}$ Stufe, welches durch das Gleichungssystem $x_{1}^{\prime}=0, x_{2}^{\prime}=0, \ldots x_{\tau}^{\prime}=0$ definiert ist. Da die Stufenzablen von $\&$ und $\& *$ eine Summe $>n$

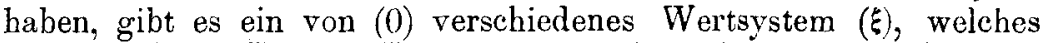
den Gebilden $\left(E\right.$ and $F^{*}$ gemeinsam ist. Es sei ( $\left.\xi^{\prime}\right)$ das entsprechende Wertsystem der neuen Variabelen. Man hat dann einerseits $f(\xi)>0$, da $(\xi)$ auf $(\xi$ liegt und von $(0)$ verschieden ist; anderseits, da $(\xi)$ auf $\xi^{*}$ liegt, $f(\xi)=-f_{2}\left(\xi_{\pi+1}^{\prime}, \xi_{\pi+2}^{\prime}, \ldots \xi_{\tau+\nu}^{\prime}\right)$, also $f(\xi) \leqq 0$. Die Annahme hat also einen Widerspruch ergeben.

Damit ist der Satz für $\pi$ bewiesen. Für v folgt er aus der Bemerkung, daß der Negativrang von $f$ der Positivrang von - $f$ ist.

Die Ausführungen dieses $\S$ dienen lediglich zur Herstellung der Verbindung mit der algebraischen Theorie und werden im Folgenden nicht benützt.

Bis hieher könnte übrigens die quadratische Form durch eine Form beliebiger Ordnung mit reellen Koeffizienten ersetzt werden.

\section{\$ 3. $\pi+v=$ Rang $\rho$ der Form $f$.} dingung :

a) Der Inbegriff derjenigen Wertsysteme (o), welche die Be-

$$
f(x+\sigma)=f(x) \text { identisch in }(x)
$$

erfüllen, werde die Spitze der Form $f$ genannt und mit $\mathfrak{S}$ bezeichnet. Man kann $\subseteq$ auch durch die Bedingung: 


$$
f(x+\sigma)=f(x)+f(\sigma) \text { identisch in }(x)
$$

definieren; die Übereinstimmung beider Definitionen beruht darauf, daß sich aus jeder von ihnen:

$$
f(\sigma)=0
$$

ergibt, indem man $(x)=(\sigma)$ einträgt. $\left.{ }^{8}\right)$

Die Spitze $\mathfrak{S}$ ist ein ebenes Gebilde. Denn vermöge der Identität:

(4) $f(x+y)=f(x)+2 f(x, y)+f(y)$ identisch in $(x)$ und $(y)$

$$
\text { - wo } f(x, y)=x_{1} f_{1}(y)+x_{2} f_{2}(y)+\cdots+x_{n} f_{n}(y), f_{i}(x)=\frac{1}{2} \frac{\partial f(x)}{\partial} \frac{x_{i}}{} \text {, }
$$

und also $f(x, y)=f(y, x)$ - erkennt man eine dritte Definition von $\subseteq$, nämlich durch die Bedingung:

$$
f(x, \sigma)=0 \text { identisch in }(x),
$$

d. i. durch das linearhomogene Gleichungssystem:

$$
f_{1}(\sigma)=0, f_{2}(\sigma)=0, \ldots f_{n}(\sigma)=0 .
$$

Ist nun $\subseteq$ von der Stufe $n-p$, also das Gleichungssystem (6) vom Range $p$, so heibt $p$ der Rang der Form $f .{ }^{9}$ )

b) Beweis fui $r+v>p$. Es kommt blok daranf an, ein ebenes Gebilde $p-\pi^{\text {ter }}$ Stufe zu konstruieren ( $C^{\prime \prime}$ unten), auf welchem durchaus $f<0$ mit alleiniger Ausnahme des Wertsystems (0).

Es sei E ein ebenes Gebilde möglichst hoher $\left(\pi^{\text {ter }}\right)$ Stufe von der Beschaffenheit, daß:

$$
f(\varepsilon)>0 \text { für alle }(\varepsilon) \text { von }(E \text { außer }(0) \text {. }
$$

Der Inbegriff aller Wertsysteme $\left(\varepsilon^{\prime}\right)$, welche die Bedingung:

erfüllen, werde das $z \mathfrak{u}$ E in Bezug aufdie Form $f$ polarreziproke Gebilde genannt und mit \&' bezeichnet.

$\mathbb{C}^{\prime}$ enthält alle Wertsysteme (o) derSpitze $\circlearrowleft$; denn für diese gilt (2), umsomehr also :

$$
f(\varepsilon+\sigma)=f(\varepsilon)+f(\sigma) \text { für alle }(\xi) \text { ron } \xi \text {. }
$$

(E) hat mit seinem polarreziproken Gebilde $\mathbb{E}$ kein Wertsystem au 0 er $(0)$ gemein; denn für $\left(\varepsilon^{\prime}\right)=(\xi)$ ergibt $(8)$ : $f(s)=0$ im Widerspruch mit $(7)$.

${ }^{8}$ ) Oder z. B. $(x)=(0)$ in (1) und $(x)=-(\sigma)$ in (2).

9) Die Bedeutung von $p$ als der kleinsten Zabl von Veränderlichen, auf die man $f$ durch linearhomogene Transformation mit nichtverschwindender. Determinante bringen kann, ergibt sich von hier aus durch die folgende einfache Bemerkung. Ist $(x)=(\xi) X+\cdots+(\zeta) Z+(\rho) R+\cdots+(\tau) T$ eine solche Transformation, so ist dafür, dab $f(() X+\cdots+(\tau) T)$ von den Variabelen $R \ldots T$ unabhăngig wird, offenbar notwendig und hinreichend, daß $(\rho), \ldots(\tau)$ die Eigenschaft (1) haben, d. h. zu $\mathfrak{S}$ gehören. Ihre Anzahl ist also im besten Falle gleich der Stufe von $\subseteq$, d. i. $n-\rho$; die Anzabl der verbleibenden Variabelen $X, \ldots Z$ also p. 
Das zu E polarreziproke Gebilde E' istein ebenes Gebilde der Stufe $n-\pi$. Denn vermöge der Identität (4) erkennt man eine zweite Definition von $\mathbb{E}^{\prime}$, nämlich durch die Bedingung :

$$
f\left(\varepsilon, s^{\prime}\right)=0 \text { für alle }(\varepsilon) \text { aus }(E ;
$$

damit aber diese Gleichung für alle $(s)$ von $\&$ bestehe, ist hinreichend, daß sie für $\pi$ linearunabhängige $(\varepsilon)$ aus $\mathbb{E}$ erfüllt sei; mithin ist $(\vec{F}$ durch ein System von $\pi$ linearhomogenen Gleichungen bestimmt, ist also ein ebenes Gebilde von mindestens $n-\pi$ ter Stufe. Anderseits ist, da $F$ und $F^{\prime}$ kein Wertsystem außer (0) gemein haben, die Summe ihrer Stufenzahlen höchstens gleich $n$, also die Stufenzahl ron (F' höchstens $n-\pi$.

Es is t $f\left(\varepsilon^{\prime}\right) \leq 0$ für alle $\left(\varepsilon^{\prime}\right)$ aus $\left(\xi^{\prime}\right.$.

Beweis: Gesetzt, es gebe ein Wertsystem $\left(\varepsilon^{\prime}\right)$ auf $\left(\xi^{\prime}\right.$, für welches $f\left(\varepsilon^{\prime}\right)>0$. Dann durchläuft

$$
\left(\gamma_{1}\right)=\left(\varepsilon^{\prime}\right) \lambda+(\varepsilon)
$$

wenn $\lambda$ alle reellen Zahlen und $(\varepsilon)$ alle Wertsysteme von $\&$ durchläuft, ein ebenes Gebilde $\pi+1^{\text {ter }}$ Stufe, da $\mathbb{E}^{-}$und $\mathbb{E}^{\prime}$ kein Wertsystem außer (0) gemein haben. Nach (8) ist aber :

mithin :

$$
f\left(r_{1}\right)=f\left(\left(\varepsilon^{\prime}\right) \lambda\right)+f(\varepsilon)
$$

$$
f\left(r_{i}\right)>0 \text { für alle }\left(r_{i}\right) \text { auber }(0),
$$

was jedoch bei einem Gebilde von höherer als $\pi^{\text {ter }}$ Stufe nicht stattfinden kann.

$$
f\left(\varepsilon^{\prime}\right)=0 \text { gilt nur für diejenigen }\left(\varepsilon^{\prime}\right) \text { von (E', welche }
$$
der Spitze $\subseteq$ angehören.

Beweis: Sei $(\omega)$ ein Wertsystem von $\mathfrak{E}^{\prime}$, für welches $f(\omega)=0$. Dann ist auch

$$
\left(\eta^{\prime}\right)=(\omega)+\lambda\left(\varepsilon^{\prime}\right)
$$

für jede reelle Zahl $\lambda$ und jedes $\left(\varepsilon^{\prime}\right)$ von $\varepsilon^{\prime}$ ein Wertsystem von $\mathscr{E}$, mithin:

$$
f\left(r^{\prime}\right) \leqq 0 \text {. }
$$

Nach der Identität (4) ergibt dies für jedes ( $\left.\varepsilon^{\prime}\right)$ aus $\varepsilon^{\prime}$ :

$$
2 \lambda f\left(\omega, \varepsilon^{\prime}\right)+\lambda^{2} f\left(\varepsilon^{\prime}\right) \leqq 0 \text { für alle } \lambda \text {. }
$$

Dies ist nur möglich, wenn der Koeffizient von $\lambda$ verschwindet, daher ist:

$$
f\left(\omega, \varepsilon^{\prime}\right)=0 \text { für alle }\left(\varepsilon^{\prime}\right) \text { von } \varepsilon^{\prime} .
$$

Anderseits wird die Tatsache, daß $(\omega)$ auf $\left(\&^{\prime}\right.$ liegt, nach (9) ausgedrückt durch :

$$
f(\omega, \varepsilon)=0 \text { für alle }(\varepsilon) \text { von } \varepsilon
$$


Aus ( $\alpha$ ) und ( $\beta$ ) folgt aber:

$f\left(\omega, \varepsilon+\varepsilon^{\prime}\right)=0$ für jedes $(\varepsilon)$ aus $\left(\xi\right.$ und jedes $\left(\varepsilon^{\prime}\right)$ aus $\varepsilon^{\prime}$, d. i. :

$$
f(\omega, x)=0 \text { identisch in }(x) \text {. }
$$

Nach der Definition (5) von $\varsigma$ gehört also $(\omega)$ zu $\Subset$.

Es sei (5) ein ebenes Gebilde möglichst hoher Stufe, welches in $\mathbb{F}^{\prime}$ enthalten ist und mit $\mathfrak{S}$ kein Wertsystem a $b$ er $(0)$ gemein hat.

Dann ist $f\left(\varepsilon^{\prime \prime}\right)<0$ für alle $\left(\varepsilon^{\prime \prime}\right)$ von $\varepsilon^{\prime \prime}$ außer $(0)$. Die Stufenzahl von $\mathscr{E}^{\prime \prime}$ ist die Differenz aus den Stufenzahlen von $\mathscr{E}^{\prime}$ und $\mathbb{S}$, beträgt also $p-\pi$. Mithin ist $p-\pi \leq \nu$, d. i.:

c) Beweis für $\pi+\nu \leq p$.

$$
\pi+v \geq p \text {. }
$$

Es sei $\bar{E}$ ein ebenesGebilde möglichst hoher (vter) Stufe von der Beschaffenbeit, daB:

$$
f(\bar{\varepsilon})<0 \text { für alle }(\bar{\varepsilon}) \text { von } \overline{(} \text { außer }(0) \text {. }
$$

Dann haben zuvörderst wegen (7) und (10) die Gebilde $(5$ und $\bar{\varepsilon}$ kein Wertsystem außer $(0)$ gemein. Ist ferner $(\varepsilon)$ ein beliebiges Wertsystem aus $(\vec{\xi},(\ddot{\xi})$ ein beliebiges aus $\bar{\xi}$, so kann $(\xi+\bar{\varepsilon})$ nie zu $\subseteq$ gehören, es sei denn das Wertsystem $(0)$; andernfalls nämlich würde (1) für $(\sigma)=(\varepsilon)+(\xi)$ und $(x)=-(\Xi)$ ergeben: $f(\varepsilon)=f^{\prime}(\bar{\varepsilon})$. Demnach ist die Summe der Stufenzahlen von $\mathbb{E}, \bar{E}$ und $\subseteq$ nicht gröber als $n, \pi+\nu+(n-\rho) \leqq n$, d. i.:

$$
\pi+\nu \leq p \text {. }
$$

Die Zusammenfassung von $b$ ) und $c$ ) ergibtschliebli ch: $\pi+v=p$. knüpfen.

An diese Betrachtungen möchte ich noch zwei Bemerkungen

d) Umkehru-ng zu $\S 2$.

Da $\rho-\pi=\nu$ ist, hat (F" die Stufenzahl $v$, und dieser Um. stand läßt sich zur Umkehrung des in $\$ 2$ erơrterten Satzes benützen: Die gegebene Form $f$ habe den Positivrang $\pi$ und den Negativrang $\vee ;$ es soll gezeigt werden, daß sich $f$ in die 1. c. näher erklärte Gestalt $f_{1}-f_{2}$ setzen läßt.

Zu dem Ende wähle man ein beliebiges ebenes Gebilde (E von der $\pi^{\text {ten }}$ Stufe, auf welchem mit alleiniger Ausnahme des Wertsystems $(0)$ stets $f>0$ ist, und konstruiere ein ( $($ '" wie oben $(b)$. Haben nun $(5)$ und $\Phi$ die frühere Bedeutung, und bezeichnen ( $\varepsilon$ ), $\left(\varepsilon^{\prime}\right),\left(\varepsilon^{\prime \prime}\right),(\sigma)$ Wertsysteme aus resp. $\left(E, \mathbb{E}^{\prime}, \mathbb{E}^{\prime \prime}\right.$, S, so kann jedes Wertsystem $(x)$ auf eine and nur eine Weise in die Gestalt 
$(x)=\left(\varepsilon+\varepsilon^{\prime}\right)$, weiter dieses $\left(\varepsilon^{\prime}\right)$ auf eine und nur eine Weise in die Gestalt $\left(\varepsilon^{\prime}\right)=\left(\varepsilon^{\prime \prime}+\sigma\right)$ gesetzt werden, und man hat: $f(x)=$ $=f\left(\varepsilon+\varepsilon^{\prime}\right)=f(\varepsilon)+f\left(\varepsilon^{\prime}\right)=f(\varepsilon)+f\left(\varepsilon^{\prime \prime}+\sigma\right)=f(\varepsilon)+f\left(\varepsilon^{\prime \prime}\right)$, d. i.

$$
f(x)=f(\varepsilon)+f\left(\varepsilon^{\prime \prime}\right) \text {, }
$$

womit die gew ünschte Darstellung im wesentlichen erreicht ist. Haben nämlich (F, (F" und 5 die Parameterdarstellungen:

$$
\begin{aligned}
(\varepsilon) & =(\xi)_{1} x_{1}^{\prime}+\cdots+(\xi)_{\pi} x_{\pi}^{\prime}, \\
\left(\varepsilon^{\prime \prime}\right) & =(\xi)_{\pi+1} x_{\pi+1}^{\prime}+\cdots+(\xi)_{\pi+\nu} x_{\pi+v}^{\prime}, \\
(\sigma) & =(\xi)_{\pi+\nu+1} x_{\pi+\nu+1}^{\prime}+\cdots+(\xi)_{n} x_{n}^{\prime},
\end{aligned}
$$

mit den Parametern $x_{1}^{\prime} \ldots x_{n}^{\prime}$, so ist

$$
(x)=(\xi)_{1} x_{1}^{\prime}+\cdots+(\xi)_{n} x_{n}^{\prime}
$$

eine Transformation mit nichtverschwindender Determinante (vgl. c), und wenn

$$
\begin{aligned}
f(\varepsilon) & =f\left((\xi)_{1} x_{1}^{\prime}+\cdots+(\xi)_{\pi} x_{\pi}^{\prime}\right)=f_{1}\left(x_{1}^{\prime}, \cdots x_{\pi}^{\prime}\right), \\
f\left(\xi^{\prime \prime}\right) & =f\left((\xi)_{\tau+1} x_{x+1}^{\prime}+\cdots+(\xi)_{\pi+v} x_{\pi+\nu}^{\prime}\right)=-f_{2}\left(x_{\pi+1}^{\prime}, \cdots x_{\tau+\nu}^{\prime}\right)
\end{aligned}
$$

gesetzt wird, sind $f_{1}$ und $f_{2}$ positiv definite Formen ihrer $\pi$ resp. $\checkmark$ Variablen, und man hat:

$$
f(x)=f_{1}\left(x_{1}^{\prime}, \cdots x_{\pi}^{\prime}\right)-f_{2}\left(x_{\pi+1}^{\prime}, \cdots x_{\pi+\nu}^{\prime}\right) .
$$

Im folgenden wird übrigens diese Umkehrung ebensowenig benützt wie der Satz aus $\S 2$ selbst.

e) Die Zahlen $\pi^{*}$ und $\nu^{*}$.

Bisweilen (s. \& 7) empfiehlt es sich, an Stelle von $(\pi, v)$ der Form $f$ ein anderes Zahlenpaar $\left(\pi^{*}, v^{*}\right)$ zuzuordnen, welches durch folgende Bedingungen definiert ist: Es gebe ein ebenes Gebilde $\pi^{* \text { ter }}$, aber keines $\pi^{*}+1^{\text {ter }}$ Stufe, auf welchem durchaus $f \geq 0$, und es gebe ein ebenes Gebilde $v^{* \text { ter }}$, aber keines $\nu^{*}+1^{\text {ter }} \widehat{\text { Stufe, }}$ auf welchem durchaus $f \leq 0$.

Diese neuen Zahlen hängen mit den alten in der Weise zusammen, daß:

$$
\begin{aligned}
& \pi^{*}=\pi+(n-p)=n-\nu, \\
& \nu^{*}=\nu+(n-p)=n-\pi .
\end{aligned}
$$

Beweis: Es sei $\left(5\right.$ ein ebenes Gebilde $\pi^{\text {ter }}$ Stufe, auf welchem mit alleiniger Ausnahme des Wertsystems (0) durchaus $f>0$; ferner sei $\mathfrak{S}$ die Spitze der Form $f$; dann haben $\mathbb{E}$ und $\mathbb{S}$ nur das Wertsystem (0) gemein. Durchläuft nun ( $\varepsilon$ ) alle Wertsysteme von $(\mathbb{E}$ und $(\sigma)$ alle Wertsysteme von $\subseteq$, so durchläuft $(\varepsilon+\sigma)$ ein ebenes Gebilde $\pi+(n-\rho)^{\text {tex }}$ Stufe, auf welchem wegen (1) durchaus $f \geq 0$. Mithin ist $\pi^{*} \geq \pi+(n-p)$. 
Anderseits sei $\mathbb{F}^{*}$ ein ebenes Gebilde $\pi^{\text {ter }}$ Stufe, auf welchem durchaus $f \geq 0$, $\bar{E}$ ein ebenes Gebilde ${ }^{\text {ter }}$ Stufe, auf welchem mit alleiniger Ausnahme des Wertsystems (0) durchaus $f<0$; dann haben (5* und $\bar{E}$ nur das Wertsystem (0) gemein, da $f$ nicht zugleich $>0$ und $<0$ sein kann. Folglich beträgt die Summe $\pi^{*}+v \overline{\overline{i h}}$ rer Stufenzahlen höchstens $n$, so dab $\pi^{*} \leq n-v$.

Für $v^{*}$ wird der Beweis analog geführt, oder auch auf den für $\pi^{*}$ zurückgeführt, indem man $f$ durch $-f$ ersetzt.

\section{$\$$ 4. Die Funktionen $\pi(t)$ und $v(t)$; Hilfssatz über den Rang $f(t)$.}

Um den Positiv- und Negativrang der Form $f$ zu bestimmen, ist es von Vorteil, $f$ als Element der Formenschar mit dem reellen Parameter $t$ :

$$
f(x)-t F(x)
$$

wo $F$ irgend eine positiv definite Form bedeutet, zu betrachten und zuvörderst den Positivrang $\pi(t)$ und Negativrang $\nu(t)$ der Form $f-t F$ als Funktionen von $t$ zu studieren.

Die Summe dieser beiden Funktionen ist nach $\S 3$ stets gleich dem Range $p(t)$ der Form $f-t F$. Dieser ist nach der Definition in $\S 3, a)$ gleich dem Range des Gleichungssystems:

(11) $f_{1}(x)-t F_{1}(x)=0, f_{2}(x)-t F_{2}(x)=0, \ldots f_{n}(x)-t F_{n}(x)=0$, wo $F_{i}=\frac{1}{2} \frac{\partial F}{\partial x_{i}} \cdot$ Es ist also stets $\rho(t) \leq n$. Die Werte $t$ ferner für welche $\rho(t)<n$ ist, sind die reellen Wurzeln der Gleichung:

$$
\left|\begin{array}{c}
a_{11}-t A_{11}, a_{12}-t A_{12}, \cdots a_{1 n}-t A_{1 n} \\
a_{21}-t A_{21}, a_{22}-t A_{22}, \cdots a_{2 n}-t A_{2 n} \\
\ldots \ldots \ldots \ldots \ldots \ldots \cdots \cdots \cdots \\
a_{n 1}-t A_{n 1}, a_{n 2}-t A_{n 2}, \cdots a_{n n}-t A_{n n}
\end{array}\right|=0
$$

wo $a_{i k}=\frac{1}{2} \frac{\partial^{2} f}{\partial x_{i} \partial x_{k}}, A_{i k}=\frac{1}{2} \frac{\partial^{2} F^{i}}{\partial x_{i} \partial x_{k}}$ und also $a_{i k}=a_{k i}, A_{i k}=$ $=A_{k i}$; sie sind daher nur in endlicher ${ }^{10}$ ) Zahl vorhanden; insbesondere in der Zahl 0, falls (12) keine reellen Wurzeln haben sollte.

Es sei $t=a$ eine $\alpha^{\text {fache }}$ reelle Wurzel der Gleichung (12), wobei $\alpha>0$. Bildet man das Gleichungssystem (11) für $t=a$ :

$$
f_{1}(x)=a F_{1}(x), f_{2}(x)=a F_{2}(x) \ldots f_{n}(x)=a F_{n}(x),
$$

so hängt die Anzahl $n-p(a)$ der linearunabhängigen Lösungen dieses Gleichungssystems aufs engste mit der Multiplizität $\alpha$

10) Der Grad der Gleichung (12) beträgt $n$, da $F$ definit; es genügt aber für das Folgende, festzuhalten, daß er $\leq n$, und daß (12) keine Identität. 
der Wurzel a zusammen. Die eine Hälfte dieses Zusammen'hanges enthält der

$$
\text { Hilfssatz: } a \geq n-\rho(a) \text {. }
$$

Beweis: Ės seien $(\mathfrak{a}),\left(\mathfrak{a}^{\prime}\right), \ldots n-\rho(a)$ linearunabhängige Lösungen von $(13)$, und $(\mathfrak{r}),\left(\mathfrak{x}^{\prime}\right), \ldots p(a)$ derart gew ihlte Wertsysteme, $d a b$ in $(\mathfrak{a}),\left(\mathfrak{a}^{\prime}\right) \ldots(\mathfrak{x}),\left(\mathfrak{x}^{\prime}\right), \ldots n$ linearunabhängige Wertsysteme vorliegen. Dann ist :

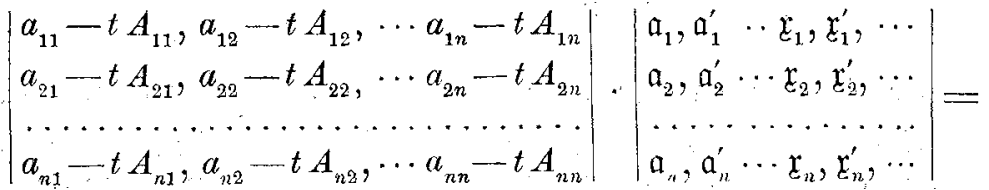

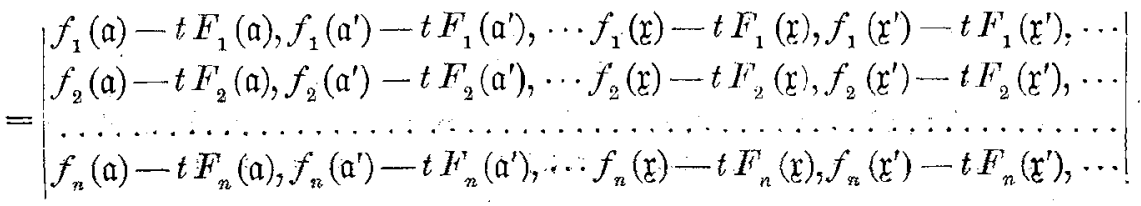

Trägt man in die rechtsstehende Determinante die durch (13) gegebenen Werte:

$$
\begin{aligned}
& f_{1}(\mathfrak{a})=a F_{1}(\mathfrak{a}), f_{2}(\mathfrak{a})=a F_{2}(\mathfrak{a}), \cdots f_{n}(\mathfrak{a})=a F_{n}(\mathfrak{a}), \\
& f_{1}\left(\mathfrak{a}^{\prime}\right)=a F_{1}\left(\mathfrak{a}^{\prime}\right), f_{2}\left(\mathfrak{a}^{\prime}\right)=a F_{2}\left(\mathfrak{a}^{\prime}\right), \cdots f_{n}^{\prime}\left(\mathfrak{a}^{\prime}\right)=a F_{n}^{\prime}\left(\mathfrak{a}^{\prime}\right),
\end{aligned}
$$

ein, so erhält jede der ersten $n-p(a)$ Vertikalreihen den Faktor $a-t$. Folglich ist die erste Determinante linker Hand, d. i. die linke Seite der Gleichung (12), durch die $n-p(a)^{\text {te }}$ Potenz dieses Faktors teilbar.

Im folgenden werden von der Funktion $p(t)$ blob die in diesem $§$ besprochenen Eigenschaften benützt.

\section{\$5. Die vier charakteristischen Eigenschaften der Funktionen $\tau=(t)$ und $v(t)$.}

Die Funktionen $\pi(t)$ und $\nu(t)$ sind eindeutig mit reellen ${ }^{11}$ ) Werten definiert für alle reellen Werte von $t$ und besitzen die folgenden vier Eigenschaften, ${ }^{12}$ ) welche sich in $\$ 6$ auch als charakteristisch erweisen :werden.

I. Bezeichnet man ibre Summe mit $p(t)$, so ist :

$$
n-\tau \leqq p(t) \leqq n
$$

wenn $t$ eine fache Wurzel der Gleichting (12) ist; und zwar auch im Falle $\tau=0$.

i1) DaB die Funktionen nur ganzzahliger, oder daß sie nur endlich vieler verschiedener Worto fahig sind, wird in das System charakteristischer Eigenschaften nich aufgenommen.

1.2) Davon sind II, III., IV. geometrisch evident. 
Diese Eigenschaft ist in $\S 4$ vollständig nachgewiesen worden.

II. Sie sind monoton: aus $t_{1}<t_{2}$ folgt $\pi\left(t_{1}\right)>\pi_{(}\left(t_{2}\right), v\left(t_{1}\right)<v\left(t_{2}\right)$. Denn es ist: $\left(f-t_{1} F\right)-\left(f-t_{2} F\right)=\left(t_{2}-t_{1}\right) \bar{F}>0$ für $(x)==(0)$.

Den Eigenschaften III und IV werde die folgende Erörterúng vorausgeschickt, welche den Umstand zur Geltung bringt, daf die quadratischen Formen stetige Funktionen sind:

Da proportionale Wertsysteme der $(x)$ Funktionswerte rom selben Vorzeichen liefern, genügt es; statt eines ebenen Gebildes (E) blof seinen Schnitt $E$ mit der aus den reellen Lösungen der Gleichung $F(x)=1$ bestehenden Mannigfaltigkeit $\mathrm{zu}$ betrachten. Nun ist diese Mannigfaltigkeit $F=1$ im Endlichen gelegen ${ }^{13}$ ) und. abgeschlossen; das Gleiche gilt daher für $E$. Da anderseits $f$ stetig ist, gibt es unter den Werten, welche $f$ auf $E$ annimmt, einen größten und einen kleinsten. Wir bezeichnen dieselben mit:

$$
\max \text { (\&) und min. (E). }
$$

Nunmehr ist eine Aussage wie $f-t F>0$ auf $\left(E\right.$ außer $(0)^{\text {" }}$ gleichbedeutend mit "min. (E) $>t$, ebenso eine Aussage wie $f-t F<0$ auf $\mathbb{E}$ auber $(0)^{\text {" }}$ gleichbedeutend mit "max. (\&) $<t^{*}$, desgleichen eine Aussage wie "f $f-t F>0$ auf $\mathbb{F}$ " gleichbedeutend

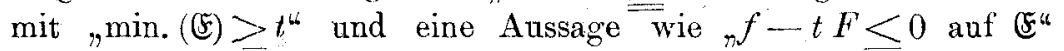
gleichbedeutend mit "max. (ङ) $<t^{\sharp}$.

Dies vorweggenommen, fahren wir in der Aufzählung der vier Eigenschaften fort.

III. $\pi(+\infty)=0, v(-\infty)=0$. Will sagen: es gibt einen Wert $g$ und einen Wert $h$, sodaß für $t \geq g$ stets $\pi(t)=0$ und für $t<h$ stets $\vee(t)=0$.

Beweis: Man braucht nur $g=\max .\left(\aleph_{n}\right)$ za setzen, wo $\aleph_{n}$ das einzig existierende ebene Gebilde $n^{\text {ter }}$ Stufe, nämlich den. Inbegriff aller reellen Wertsysteme, bedeutet. In der Tat, für $t>\max .\left(\mathscr{\mho}_{n}\right)$ ist $f-t F<0$ auf $\vec{E}_{n}$, mithin $\pi(t)=0$. - Damit ist die eine Hälfte der $\overline{\overline{B e}}$ hauptung erwiesen. Die andere Hälfte folgt aus dieser,

13) Dies ist auch ohne Inanspruchnahme des algebraischen Hilfsmittels der linearen Transformation leicht einzusehen. Betrachtet man die Funktion $\boldsymbol{F}^{\prime}(x)$ auf derjenigen Mannigfaltigkeit, welche aus allen reellen Lösungen der Gleichung: $x_{1}^{2}+x_{2}^{2}+\ldots+x_{n}^{2}=1$ besteht, so gibt. es unter ihren Werten einen kleinston Fo; denn die Mannigfaltigkeit $x_{1}^{2}+x_{2}^{2}+\ldots+x_{n}^{2}=1$ ist im Endlichen gelegen und abgeschlossen, die Funktion $F(x)$ stetig. Da aber $F(x)$ positiv definit, ist $F_{0}>0$. Nun ist $F(x) \geqq F_{0}$ auf der Mannigfaltigkeit $x_{1}^{2}+x_{2}^{2}+\ldots+x_{n}^{2}=1$, mithin $F(x) \geqq F_{0} \cdot\left(x_{1}^{2}+x_{2}^{2}+\ldots+x_{n}^{2}\right)$ für alle möglichen Wertsysteme $(x)$. $f^{\prime}(x)=1$ kann daher nur stathaben für: $F_{0} \cdot\left(x_{1}^{2}+x_{2}^{2}+\ldots+x_{n}^{2}\right) \leqq 1$ oder $x_{1}^{2}+x_{2}^{2}+\cdots+x_{n}^{2} \leqq \frac{1}{F_{0}}$. 
indem man $f$ durch $-f$ ersetzt, oder kann auch analog bewiesen werden; hier genügt $h=\min .\left(\tilde{\mho}_{n}\right)$ der gestellten Forderung.

IV. Jede Stelle $t_{0}$ besitzt eine rechtsseitige Umgebung $t_{0}<t<t_{0}+\delta$, in welcher durchaus $\pi(t)>\pi\left(t_{0}\right)$, und eine linksseitige Umgebung $t_{0}-\delta^{\prime}<t<t_{0}$, in welcher durchaus $v(t) \geq v\left(t_{0}\right)$. Beweis (für $\pi(t)$ ): Es sei $\mathbb{E}$ ein ebenes Gebilde von der Stufe $\pi\left(t_{0}\right)$, auf welchem mit alleiniger Ausnahme des Wertsystems (0) durchaus $f-t_{0} F>0$. Dann ist min. (E) $>t_{0}$, und man kann min. (E) $=t_{0}+\delta$ setzen, wo $\delta>0$. Für $t<t_{0}+\delta$, d. i. $t<\min$. (E), ist nun: $f-t F>0$ auf $(\mathbb{F}$ außer $(0)$, mithin $\pi(t)$ mindestens gleich der Stufenzahl $\pi\left(t_{0}\right)$ von $E: \pi(t) \geq \pi\left(t_{0}\right)$ für $t<t_{0}+\delta$. - (Berücksichtigt man für die rechtsseitige Umgebung noch Eigenschaft II, so kann man sogar genauer sagen: $\pi(t) \geq \pi\left(t_{0}\right)$ für $t<t_{0}$ und $\pi(t)=\pi\left(t_{0}\right)$ für $t_{0} \leqq t<t_{0}+\delta$. Um aber alles ausscheiden, was neben Eigenschaft $\overline{\bar{I}}$ [ überflüssig, wurde die obige Formulierung der Eigenschaft IV vorgezogen.)

\section{$\$$ 6. Der Verlauf der Funktionen $\pi(t)$ und $\vee(t)$, ermittelt aus ihren vier charakteristischen Eigenschaften.}

Durch die Eigenschaften I-IV sind nun die Funktionen $\pi(t)$ und $\nu(t)$ völlig bestimmt, sobald die reellen Wurzeln der Gleichung (12) und ihre Multiplizitäten bekannt sind. In der Tat werden wir jetzt die Bestimmung der Funktionen durchführen, ohne auf ihre Bedeutung für die quadratische Form

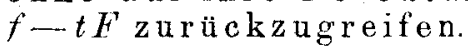

Ist $t_{0}$ ein Wert, für den $\rho\left(t_{0}\right)=n$ ist, so gibt es eine beidseitige Umgebung ron $t_{0}$, in welcher $\pi(t)$ und $v(t)$ konstant sind. Dies folgt aus I, II, IV durch die folgenden Schlüsse. Es gibt nach II und IV eine beidseitige Ungebung von $t_{0}$, in welcher durchaus $\pi(t) \geq \pi\left(t_{0}\right), v(t) \geq v\left(t_{0}\right)$, mithin $\rho(t) \geqq \rho\left(t_{0}\right)$ ist. Da aber nach Voraussetzung $\rho\left(t_{0}\right)=n$ und nach $\mathrm{I} \rho(t) \lesssim n$, sind die Zeichen $>$ ungültig und man hat $\pi(t)=\pi\left(t_{0}\right), v(t) \equiv v\left(t_{0}\right)$, w. z. b. w.

$p(t)=n$ findet aber nach I statt für alle Werte $t$, welche der Gleichung (12) nich t genügen. Sind also

$$
a<b<c<\cdots<d<e
$$

die von einander verschiedenen reellen Wurzeln der Gleichung (12), und betrachtet man die Intervalle

$$
(-\infty, a),(a, b),(b, c), \cdots(d, e),(e,+\infty) \text {, }
$$

bezw., falls (12) keine reellen Wurzeln haben sollte, das eine Intervall $(-\infty,+\infty)$, so ist im Innern dieser Intervalle durchaus $\rho(t)=n$.

Diese beiden Tatsachen gestatten den Schluß, daß im Innern eines jeden dieser Intervalle $\pi(t)$ and $\nu(t)$ konstant sind. Denn sind $t_{1}<t_{2}$ irgend zwei Werte im Innern eines und desselben Intervalls, so betrachte man die obere Grenze $t_{0}$ derjenigen unter- 
halb $t_{2}$ gelegenen Werte $t$, für welche $\pi(t)=\pi\left(t_{1}\right)$. Dann liegt auch $t_{0}$ im Innern des Intervalls, und es ist daher $p\left(t_{0}\right)=n$. Folglich gibt es um $t_{0}$ eine beidseitige Umgebung, in welcher $\pi(t)$ konstant $=\pi\left(t_{0}\right)$ ist. Dann muß aber nach dem Begriffe der oberen Grenze einerseits $\pi\left(t_{0}\right)=\pi\left(t_{1}\right)$, anderseits $t_{0}=t_{2}$ sein, sodaß sich $\pi\left(t_{2}\right)=\pi\left(t_{1}\right)$ erweist. Da ferner $\rho\left(t_{2}\right)=\rho\left(t_{1}\right)=n$, ist auch $\vee\left(t_{2}\right)=$ $=v\left(t_{1}\right)$.

Die Werte von $\pi(t)$ und $\nu(t)$ im Innern der beiden Intervalle $(-\infty, a)$ und $(e,+\infty)$ ergeben sich aus III in Verbindung mit der Tatsache $\rho(t)=n$. Und diese Werte zeigen noch, daß jedenfalls diese beiden äußersten Intervalle von einander verschieden sind, also die Gleichung (12) mindestens eine reelle Wurzel besitzt.

Betrachten wir endlich zwei aufeinanderfolgende Intervalle, so hat im Grenzpunkte zwischen ihnen wegen IV und II $\pi(t)$ denselben Wert wie im zweiten, $\nu(t)$ denselben Wert wie im ersten von ihnen.

Hiernach kann nun der Verlauf der Funktionen $\pi(t)$ und $\checkmark(t)$ dargestellt werden durch das folgende Schema, in welchem $\mathfrak{a}, \mathfrak{b}, \mathfrak{c}, \ldots \mathfrak{b}, \mathfrak{e}$ reelle Zahlen bedeuten.

\begin{tabular}{|c|c|c|c|c|c|c|c|}
\hline Für & $t<a$ & $t=a$ & $a<t<b$ & $t=b$ & $b<t<c$ & $t=c$ & $\ldots$ \\
\hline ist $\pi(t)=$ & $n$ & $n-\mathfrak{a}$ & $n-\mathfrak{a}$ & $n-\mathfrak{a}-\mathfrak{b}$ & $n-\mathfrak{a}-\mathfrak{b}$ & $n-\mathfrak{a}-\mathfrak{b}-\mathfrak{c}$ & $\ldots$ \\
ist $\nu(t)=$ & 0 & 0 & $\mathfrak{a}$ & $\mathfrak{a}$ & $\mathfrak{a}+\mathfrak{b}$ & $\mathfrak{a}+\mathfrak{b}$ & $\cdots$ \\
\hline
\end{tabular}

\begin{tabular}{|c|c|c|}
\hline$\cdots$ & $t==e$ & $e<t$ \\
\hline$\cdot$ & $\begin{array}{c}n-\mathfrak{a}-\mathfrak{b}-\mathfrak{c}-\ldots-\mathfrak{b}-\mathfrak{e} \\
\mathfrak{a}+\mathfrak{b}+\mathfrak{c}+\ldots+b\end{array}$ & $\begin{array}{r}n-\mathfrak{a}-\mathfrak{b}-\mathfrak{c}-\ldots-\mathfrak{b}-\mathfrak{e} \\
\mathfrak{a}+\mathfrak{b}+\mathfrak{c}+\ldots+\mathfrak{b}+\mathfrak{e}\end{array}$ \\
\hline
\end{tabular}

Darin bestimmen sich die Werte $\mathfrak{a}, \mathfrak{b}, \mathfrak{c}, \ldots \mathfrak{b}$, $\mathfrak{e}$, wie folgt. Da nach III $\pi(+\infty)=0$ ist, erweist sich zunächst:

$$
\mathfrak{a}+\mathfrak{b}+\mathfrak{c}+\cdots+\mathfrak{b}+\mathfrak{e}=n \text {. }
$$

Ferner ergibt die Gleichung $\pi(t)+\nu(t)=\rho(t)$ für $t=a, b$, $c, \ldots d, e$ :

$$
n-\mathfrak{a}=p(a), n-\mathfrak{b}=\rho(b), \ldots n-\mathfrak{e}=p(e) .
$$

Sind nun

$$
\alpha, \beta ; \gamma, \ldots \delta, \Xi
$$

die Multiplizitäten der Wurzeln $a, b, c, \ldots d, e$, so, ist einerseits:

$$
a+\beta+\gamma+\cdots+\delta+\varepsilon \leqq n \text {, }
$$


anderseits nach I:

$$
p(a) \geq n-a, p(b) \geq n-\beta, \ldots p(e) \geq n-\varepsilon .
$$

Da nun nach (15) und (17): $\alpha \geq \mathfrak{a}, \beta \geq \mathfrak{b}, \ldots \varepsilon \geqq e$ ist, lassen sich (14) und (16) nur so vereinigen, daß

$$
\mathfrak{a}=\alpha, \mathfrak{b}=\beta, \ldots \mathfrak{e}=\varepsilon \text {. }
$$

Werden diese Werte in das Schema eingetragen, so vermag man den Verlauf der Funktionen $\pi(t)$ und $\nu(t)$ vollkommen zu übersehen, wenn die reellen Wurzeln der Gleichung (12) mit ihren Multiplizitäten bekannt sind. Man kann das Resultat in den Satz fassen:

A. $\pi(t)$ ist die Anzahl derjenigen reellen Wurzeln der Gleichung (12), welche $>t$ sind; $v(t)$ die Anzahl derjenigen, welche $<t$ sind, wobei jede Wurzel mit ihrer Multiplizitat in Rechnung zu stellen ist.

Geht man auf die Bedeutung der Funktionen $\pi(t)$ und $v(t)$ für die Form $f-t F$ zurück, so erhält man aus $A$. für $t=0$ :

B. Der Positivrang $\pi$ der Form $f$ istgleich der Anzahl der positiven, der Negativrang vgleich der Anzahl der negativen Wurzeln der Gleichung (12), jede Wurzel nach ihrer Multiplizitä gezählt.

Diese Anzahlen sind also ganz unabhängig von der Wahl der Form $F(x)$.

$\mathrm{Da}$ der Satz $B$. für jede beliebige Form $f$ gilt, umfaßt er auch den Satz $A$.

Als Nebenresultate aber ergeben sich die Sätze:

C. Die Gleichung (12) ist vom Grade $n$ und hat lauter reelle Wurzeln. Denn nach (18) und. (14) ist $\alpha+\beta+$ $\cdots+\varepsilon=n$.

D. Es ist: $\alpha=n-p(a), \beta=n-p(b), \ldots \varepsilon=n-\rho(e)$. Dieser Satz folgt aus (18) und (15), und stellt jene Verschärfung des Hilfssatzes dar, welche schon in $\$ 4$ angedeutet wurde. ${ }^{14}$ )

14) Diese Sätze $C$. und $D$, spielen in der algebraischen Theorie eine entscheidende Rolle. Und in der Tat, sind diese beiden Sätze erst bekannt, so fuibrt die folgende einfache Betrachtung ans Ziel.

Es sei $\widetilde{S}_{a}$ die Spitze der Form, $f-a F$ und (a) ein beliebiges Wertsystem von $\mathfrak{S}_{a}(\S 3, a)$, ebenso $\mathfrak{S}_{b}$ die Spitze der Form $f-b F$ und $(\mathfrak{b})$ ein beliebiges Wertsystem von $\subseteq_{b}$ u. s. w., d. h. nach Gleichung (5):

$$
f(x, \mathfrak{a})=a F^{\prime}(x, \mathfrak{a}), f(x, \mathfrak{b})=b F^{\prime}(x, \mathfrak{b}), \ldots f(x, e)=e F^{\prime}(x, e) \text { identisch in }(x) .
$$

Setzt màn in der ersten dieser Gleichungen $(x)=(\mathfrak{a})$, so ergibt sich $f(\mathfrak{a})=a F(\mathfrak{a})$. Setzt man in der ersten Gleichung $(x)=(\mathfrak{b})$, in der zweiten $(x)=(\mathfrak{a})$ und bedenkt, da $\rho$ and $b$ verschieden sind, so erhellt: $f(\mathfrak{a}, \mathfrak{b})=0$, $F(\mathfrak{a}, \mathfrak{b})=0$. Nach diesen und den analogen Gleichungen ist nun:

$$
\begin{aligned}
& f(\mathfrak{a}+\mathfrak{b}+\ldots+\mathfrak{e})=a F(\mathfrak{a})+b F(\mathfrak{b})+\ldots+e F(\mathfrak{e}), \\
& F(\mathfrak{a}+\mathfrak{b}+\ldots+\mathfrak{e})=F(\mathfrak{a})+F(\mathfrak{b})+\ldots+F^{\prime}(\mathfrak{e}) .
\end{aligned}
$$

Aus (20) ist ersichtlich, daß $(\mathfrak{a}+\mathfrak{b}+\ldots+\mathfrak{e})$ nur dann $=(0)$ sein kann, 


\section{\$ 7. Deutung der Wurzeln von (12) als Matima und Minima von Funktionswerten.}

Sind $a_{1} \geq a_{2} \geq \cdots \geq a_{n}$, sowie $b_{1} \leqq b_{2} \leqq \cdots \leqq b_{n}$ die $n$ Wurzeln der Gleichung (12), so kann man dem Satze $A$. die folgende Fassung geben :

Für $t<a_{x}$ ist: $\pi(t) \geq x$, dagegen für $t \geq a_{x}: \pi(t)<x$; für $t>b_{*}$ ist : $\nu(t) \sum_{x}$, dagegen für $t \leqq b_{x}: \nu(t)<x$.

Wir führen jetzt an Stelle von $\pi(t)$ und $\nu(t)$ die Funktionen

$$
\begin{aligned}
& \pi^{*}(t)=\pi(t)+[n-p(t)]=n-\nu(t), \\
& \nu^{*}(t)=v(t)+[n-p(t)]=n-\pi(t)
\end{aligned}
$$

ein, deren Bedeutung für die Form $f-t F$ aus $\S 3, e)$ zu entnehmen ist. Dadurch gewinnt der Satz die Gestalt:

Für $t \leqq a_{x}$ ist: $\pi^{*}(t) \geqq x$, dagegen für $t>a_{x}: \pi^{*}(t)<x$; für $t \geq b_{x}$ ist: $\nu^{*}(t) \geq x$, dagegen für $t<b_{x}$ : $\nu^{*}(t)<x$.

$\overline{\text { Nun besagt aber }}, \pi^{*}(t) \geq x^{*}$, daß ein ebenes Gebilde $(5$ der $x^{\text {ten }}$ Stufe existiert, auf welchem durchaus $f-t F \geqq 0$ oder, was dasselbe ist, min. (\$) $\geq t$ ist.

Mithin ist $a_{r}$ der größte Wert, welchen min. (E) annimmt, wenn $(\mathcal{F})$ alle ebenen Gebilde $x^{\text {ter }}$ Stufe durchläuft. Dieses Ergebnis enthält zwei Aussagen: erstens gibt es unter den Werten, welche min. (E) annimmt, wenn (E) alle ebenen Gebilde $x^{\text {ter }}$ Stufe durchläuft, einen größten, den wir mit Max. (min. $x$ ) bezeichnen wollen; zweitens besteht der

$$
\text { Satz: Max. }(\min x)=a_{x}
$$

Ebenso gibt es unter den Werten, welche max. (\&) annimmt, wenn $\&$ alle ebenen Gebilde $x^{\text {ter }}$ Stufe durchläuft, einen kleinsten, den wir mit Min. (max. $x$ ) bezeichnen, und es ist:

$$
\text { Min. }(\max x)=b_{x} \text {. }
$$

Für $x=n$, kommt man auf die bekannte Bedeutung der äußersten Wurzeln zurück. 'Von der $n^{\text {ten }}$ Stufe nämlich gibt es nur das eine ebene Gebilde $\xi_{n}$, welches aus allen möglichen reellen Wertsystemen besteht, und man hat daher:

$$
\begin{aligned}
& a_{n}=\operatorname{Max} .(\min . n)=\min .\left(\mathbb{E}_{n}\right), \\
& b_{n}=\operatorname{Min} .(\max . n)=\max .\left(\mathbb{E}_{n}\right) .
\end{aligned}
$$

wenn $(\mathfrak{a})=(0),(\mathfrak{b})=0, \ldots(\mathfrak{c})=(0)$. Wird daher $\wp_{a}$ dargestellt durch $:(\mathfrak{a})=\left(\mathfrak{a}^{\prime}\right) \mathfrak{A}^{\prime}$ $F\left(\mathfrak{a}^{\prime \prime}\right) \mathfrak{l}^{\prime \prime}+\ldots$, wo $\left(\mathfrak{a}^{\prime}\right),\left(\mathfrak{a}^{\prime \prime}\right), \ldots$ linearunabhängige Wertsysteme und $\mathfrak{A}, \mathfrak{A}^{\prime \prime}, \ldots$ anabhängigo Paxameter bedouten, ebenso $\subseteq_{b}$ durch: $(\mathfrak{b})=\left(b^{\prime}\right) \mathfrak{B}^{\prime}+\left(\mathfrak{b}^{\prime \prime}\right) \mathfrak{B}^{\prime \prime}+\ldots$ u. s. w. so müssen die Wertsysteme $\left(\mathfrak{a}^{\prime}\right),\left(\mathfrak{a}^{\prime \prime}\right), \ldots\left(\mathfrak{b}^{\prime}\right),\left(\mathfrak{b}^{\prime \prime}\right), \ldots$ linearunabhängig sein.

Nach den. Sätzen $C$. und $D$.' ist aber die Anzahl dieser Wertsysteme. gleich $n$. Man hat daher die linearhomogene Transformation mit nichtverschwindender Determinante: $(x)=\left(\mathfrak{a}^{\prime}\right) \mathfrak{\mathfrak { U } ^ { \prime }}+\left(\mathfrak{a}^{\prime \prime}\right) \mathfrak{A}^{\prime \prime}+\ldots+\left(\mathfrak{b}^{\prime}\right) \mathfrak{B}^{\prime}+\left(\mathfrak{b}^{\prime \prime}\right) \mathfrak{B}^{\prime \prime}+\ldots$, and nach (19):

$$
\begin{gathered}
f(x)=a F\left(\mathfrak{a}^{\prime} \mathfrak{X}+\mathfrak{a}^{\prime \prime} \mathfrak{Q}^{\prime \prime}+\ldots\right)+b F\left(\mathfrak{b}^{\prime} \mathfrak{B}^{\prime}+\mathfrak{b}^{\prime \prime} \mathfrak{B}^{\prime \prime}+\ldots\right)+ \\
\ldots+e F^{\prime}\left(\mathfrak{e}^{\prime}+\mathfrak{e}^{\prime \prime}\left(\mathfrak{S}^{\prime \prime}+\ldots\right) .\right.
\end{gathered}
$$

Diese Gleichung führt den Satz $B$. unmittelbar auf $\$ 2$ zurück. 